\title{
Cognitive Dysfunctions in Patients with Alcohol Dependence Syndrome in a Tertiary Hospital in Kathmandu
}

\author{
Sirjana Adhikari, ${ }^{1}$ Mita Rana, ${ }^{1}$ Suraj Shakya, ${ }^{1}$ Saroj Prasad Ojha' \\ 'Department of Psychiatry and Mental Health, Tribhuvan University Teaching Hospital, IOM, Nepal.
}

\section{ABSTRACT}

Introduction: Alcohol dependence is a chronic disorder, accompanied by neuropsychological deficits. Patients with alcohol dependence were evaluated to identify the cognitive dysfunctions.

Method: The study followed descriptive design and recruited participants $(\mathrm{N}=62)$ from inpatient services of the Department of Psychiatry and Mental Health by convenient sampling. Participants were evaluated with the Severity of Alcohol Dependence Questionnaire, Frontal Assessment Battery and PGI Memory Scale for the severity of alcohol dependence, executive impairments and memory dysfunctions respectively once they had completed alcohol detoxification.

Results: Total duration of alcohol consumption was $13.93 \pm 8.74$ years, period of dependence was $2.97 \pm 2.23$ years and $56.5 \%$ had moderate level of dependence. $33.9 \%$ of the participants had executive impairments particularly in conceptualization, programming and mental flexibility. $38 \%$ of variance in executive dysfunction was explained by illness variables, where period of alcohol dependence and education were significant predictors. $54.8 \%$ had significant memory dysfunction particularly in visual retention, remote memory, verbal retention of dissimilar pairs and delayed recall. Memory dysfunction differed significantly among the age groups $(\mathrm{F}=10.22, \mathrm{p}<0.01)$ and age was a significant predictor $(\beta=.542, \mathrm{p}<0.001) .19 \%$ variance in memory dysfunction was explained by illness variables, where duration of alcohol consumption was a significant predictor $(\beta=.485, \mathrm{p}<0.01)$.

Conclusion: Findings indicate that cognitive dysfunctions are prevalent among patients with alcohol dependence. Hence, routine neuropsychological assessment is of particular importance for early detection and remediation of underlying deficits, which completes the treatment of alcohol dependence.

Keywords: alcohol dependence; cognitive dysfunctions; executive functions; memory.

\section{INTRODUCTION}

Alcohol dependence is a cluster of cognitive, behavioral and physiological phenomena that develop after repeated use of alcohol. ${ }^{1}$ Alcohol can damage nerve cells, particularly in the prefrontal cortex, hypothalamus and cerebellum. ${ }^{2}$ The frontocerebellar circuit and the circuit of Papez, respectively involved in motor and executive abilities, and episodic memory, are mainly affected by chronic alcohol use. ${ }^{3}$ Evidence shows that executive functions principally, abstract thinking, cognitive flexibility, persistence, planning, organizing and working memory ${ }^{4,5}$ and nonverbal memories ${ }^{6}$ are

Correspondence: Sirjana Adhiakai, Department of Psychiatry and Mental Health, Tribhuvan University Teaching Hospital, IOM, Nepal, Email: siru_ad@yahoo.com, Phone: +977-9841554583. 
impaired in patients with alcohol dependence.

These cognitive impairments not only determine everyday management, but also have significant impact on the efficacy of management. Maintenance of lasting abstinence is associated with cognitive recovery in these patients. ${ }^{7}$ It therefore appears essential to identify alcohol-related cognitive impairments so that alcohol treatment can be adapted to meet their needs.

Present study is an initiative taken to identify cognitive dysfunctions particularly the executive functions and memory in patients with alcohol dependence.

\section{METHODS}

This is a descriptive study of cognitive dysfunction in patients with alcohol dependence syndrome carried out in the Department of Psychiatry and Mental Health in Tribhuvan University Teaching Hospital over a period of six months from $15^{\text {th }}$ February to $15^{\text {th }}$ August, 2015. This site was selected on the basis of convenience. All patients who were admitted for detoxification of alcohol were enrolled in the study immediately after completion of detoxification. Participants were exclude from the study if they had (a) Hamilton's Depression rating score $>16$ (b) a history of co-morbid psychiatric and medical disorders, acquired brain injury and (c) history of dependence on psychotropic substances other than alcohol.

Ethical clearance was received from the Institution Review Board. Considering the inclusion and exclusion criteria, eligible cases were recruited as the participants of the study and informed consent was obtained. There were no harms to the participants in the study. Pertinent socio-demographic information was obtained through the semi-structured interview schedule. The Severity of Alcohol Dependence Questionnaire was used to measure the severity of alcohol dependence. Neuropsychological assessments were conducted by the researcher using the Frontal Assessment Battery and the PGI Memory Scale after 72 hours of last dose of benzodiazepine used for detoxification of alcohol.

Data analysis was performed using SPSS for windows version 17. Data were expressed as mean, standard deviation wherever possible. For test of significance
ANOVA, chi-square test, correlation and regression analyses were performed.

\section{RESULTS}

A total number of 62 patients (54 Male and 8 Female) were enrolled in the study. Participant's age ranged 22 to 58 years with mean age $39.24 \pm 9.08$ years, majority were married $(80.6 \%)$, chhetri $(25.8 \%)$ by ethnicity, and $30.6 \%$ had secondary level of education with mean education of $9.16 \pm 4.64$ years.

Duration of alcohol consumption ranged 2 to 40 years with mean duration of $13.93 \pm 8.74$ years. The period of alcohol dependence ranged between 4 months to 12 years with mean duration $2.97 \pm 2.23$ years. Most of the participants $(56.5 \%)$ in the study had moderate level of alcohol dependence, followed by severe (29\%) and mild (14.5\%) physical dependency.

In the study, executive dysfunctions differed significantly with different age groups $(\chi 2=8.40$, $\mathrm{p}<0.05)$, education $(\chi 2=12.05, \mathrm{p}<0.05)$, religion $(\chi 2=8.03, p<0.05)$ and occupation $(\chi 2=9.44$, $p<0.05)$. Similarly, memory dysfunctions also differed significantly with age $(F=10.22, P<0.001)$, marital status $(\mathrm{F}=4.23, \mathrm{P}<0.05)$ and religion $(\mathrm{F}=5.06$, $\mathrm{p}<0.05)$.

\section{Executive Functions}

Mean score in the Frontal Assessment Battery (FAB) was $12.33 \pm 2.46$. In FAB, conceptualization or abstract reasoning was the most impaired domain followed by mental flexibility. Relatively, environmental autonomy was found to be preserved among the participants. Majority $(66.1 \%)$ of the participants had average level of executive functions and $33.9 \%$ had executive impairments. Executive functions were significantly correlated with age and education, total duration of alcohol consumption and period of alcohol dependence. However, these functions were insignificant with the severity of dependence. In regression analysis, total duration of alcohol intake, period and the severity of alcohol dependence accounted $38 \%$ variance in the executive functions. Age and education accounted to an additional $8 \%$ of the variance in the executive functions. In the model, period of alcohol dependence was a significant predictor of executive dysfunctions (Table 1,2). 
Adhikari et al. Cognitive Dysfunctions in Patients with Alcohol Dependence Syndrome in a Tertiary Hospital in Kathmandu

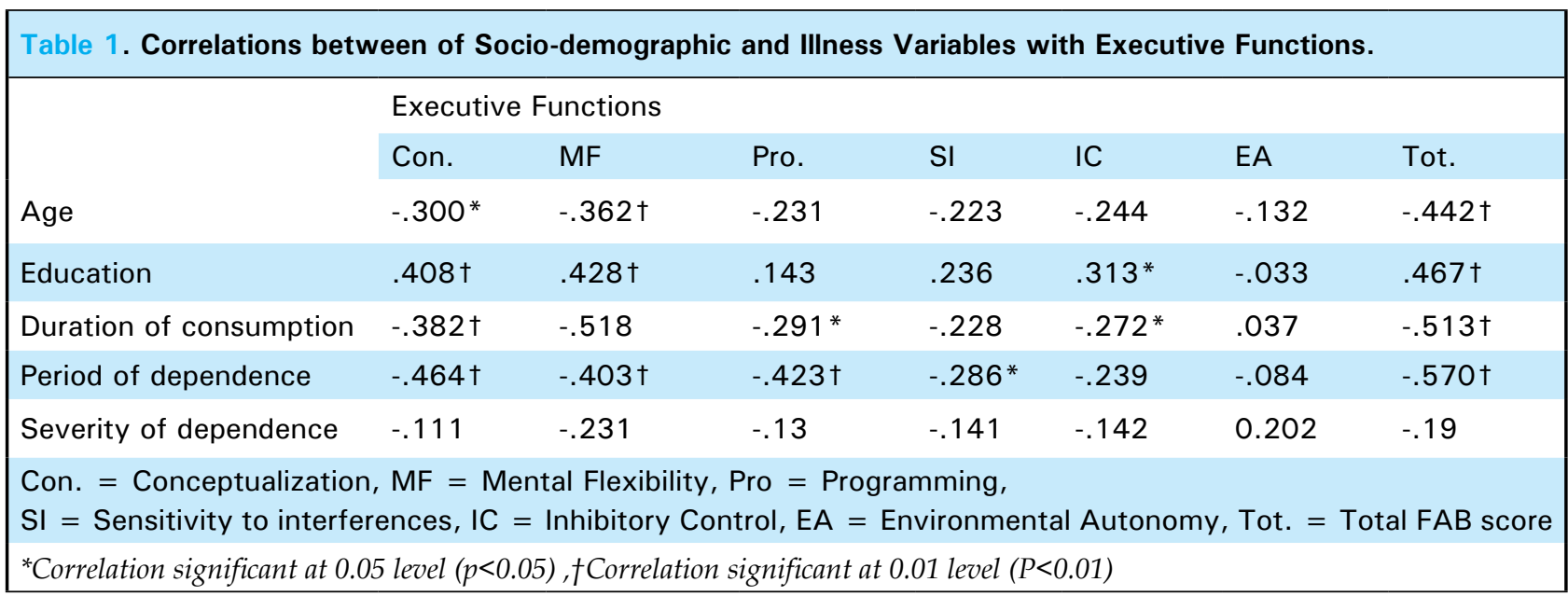

\begin{tabular}{|c|c|c|c|c|c|c|}
\hline & $\mathrm{R} 2$ & Change in $\mathrm{R} 2$ & Adjusted R2 & $\beta$ & $\mathrm{t}$ & $p$ value \\
\hline Step 1 & .385 & & .354 & & & \\
\hline Level of alcohol dependence & & & & .062 & .554 & .582 \\
\hline Duration of alcohol consumption & & & & -.301 & -2.38 & $.020 *$ \\
\hline Period of alcohol dependence & & & & -.426 & -3.39 & $.001 \dagger$ \\
\hline Step 2 & .473 & .08 & .426 & & & \\
\hline Level of alcohol dependence & & & & .051 & .480 & .633 \\
\hline Duration of alcohol consumption & & & & -.097 & -.637 & .527 \\
\hline Period of alcohol dependence & & & & -.362 & -2.99 & $.004 \dagger$ \\
\hline Age & & & & -.213 & -1.61 & .113 \\
\hline Education & & & & .284 & 2.70 & $.009+$ \\
\hline
\end{tabular}

\section{Memory}

Total dysfunctional score in the PGI Memory Scale ranged 0 to 26 with mean score $13.35 \pm 6.55$ indicating deficits in memory. Visual retention was the most impaired domain, followed by remote memory, verbal retention of dissimilar pairs, delayed recall, recognition, attention and concentration. Overall memory dysfunction was observed in $54.8 \%$ of the participants who scored 0-20 percentile score.

Dysfunctions in the recent memory, delayed recall, visual retention and visual recognition were significantly correlated with age. Similarly, total duration of alcohol consumption was positively correlated with the dysfunction in recent memory, verbal retention of similar pairs, visual retention, recognition and overall memory dysfunctions. Regression analysis revealed that alcohol related variables (total duration of alcohol intake, period and severity of alcohol dependence) accounted $19 \%$ variance in the memory dysfunctions. Duration of alcohol consumption was a significant predictor of memory dysfunctions. Age and education accounted to an additional $18 \%$ variance in memory dysfunction; age was a significant predictor of the memory dysfunction (Table 3-5). 
Adhikari et al. Cognitive Dysfunctions in Patients with Alcohol Dependence Syndrome in a Tertiary Hospital in Kathmandu

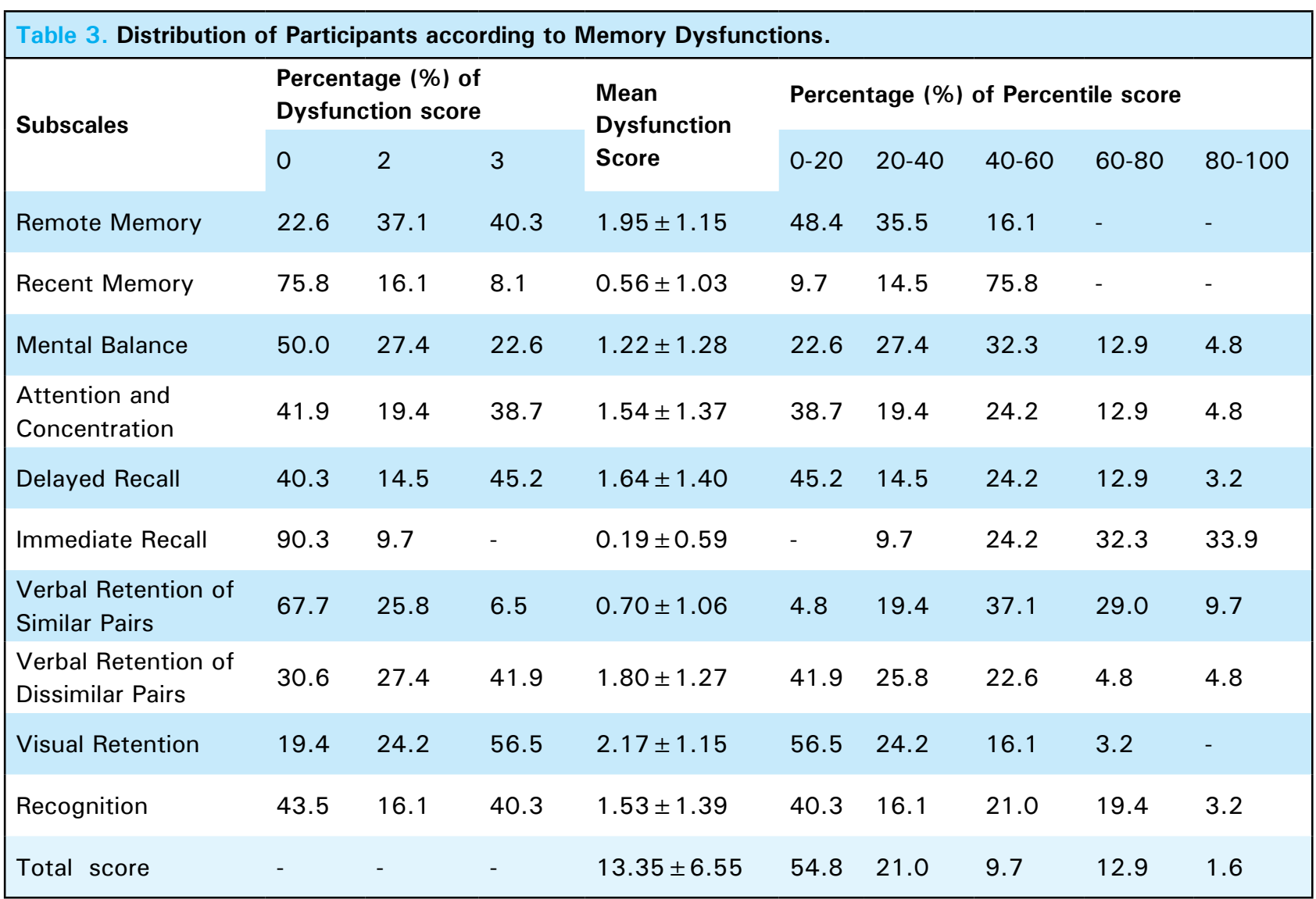

Table 4. Correlation of Socio-demographic and Illness Variables with Memory Dysfunctions.

\begin{tabular}{|c|c|c|c|c|c|}
\hline $\begin{array}{c}\text { Memory } \\
\text { Dysfunctions }\end{array}$ & Age & Education & $\begin{array}{l}\text { Duration of Alcohol } \\
\text { Consumption }\end{array}$ & $\begin{array}{c}\text { Period of } \\
\text { Dependence }\end{array}$ & $\begin{array}{l}\text { Severity of Alcohol } \\
\text { Dependence }\end{array}$ \\
\hline Remote memory & .238 & -.045 & .218 & .142 & .049 \\
\hline Recent memory & $.374 \dagger$ & -.204 & $.291 *$ & .142 & .188 \\
\hline Mental balance & .241 & .159 & .028 & -.042 & .009 \\
\hline $\begin{array}{l}\text { Attention and } \\
\text { Concentration }\end{array}$ & .485 & .040 & .178 & .020 & .043 \\
\hline Delayed recall & $.513 \dagger$ & .034 & .217 & .040 & -.054 \\
\hline Immediate recall & .058 & .131 & -.117 & -.221 & -.064 \\
\hline $\begin{array}{l}\text { Verbal retention of } \\
\text { similar pairs }\end{array}$ & .211 & -.180 & $.339 \dagger$ & .057 & -.073 \\
\hline $\begin{array}{l}\text { Verbal retention of } \\
\text { dissimilar pairs }\end{array}$ & .197 & .240 & .148 & .003 & -.021 \\
\hline Visual retention & $.301 *$ & .148 & $.335 \dagger$ & .210 & .101 \\
\hline Recognition & $.477 \dagger$ & -.162 & $.562 \dagger$ & $.484 \dagger$ & .174 \\
\hline Total score & $.593 \dagger$ & .028 & $.425 \dagger$ & .182 & .071 \\
\hline
\end{tabular}


Adhikari et al. Cognitive Dysfunctions in Patients with Alcohol Dependence Syndrome in a Tertiary Hospital in Kathmandu

\begin{tabular}{|c|c|c|c|c|c|c|}
\hline & $\mathbf{R 2}$ & Change in $\mathbf{R} 2$ & Adjusted R2 & B & $\mathbf{t}$ & $p$ value \\
\hline Step 1 & .190 & & .148 & & & \\
\hline Level of alcohol dependence & & & & -.081 & -.630 & .531 \\
\hline Duration of alcohol consumption & & & & .485 & 3.35 & $.001 \dagger$ \\
\hline Period of alcohol dependence & & & & -.058 & -.399 & .691 \\
\hline Step 2 & .376 & .18 & .320 & & & \\
\hline Level of alcohol dependence & & & & -.059 & -.515 & .609 \\
\hline Duration of alcohol consumption & & & & .134 & .803 & .425 \\
\hline Period of alcohol dependence & & & & -.012 & -.088 & .930 \\
\hline Age (years) & & & & .542 & 3.77 & $.000 \ddagger$ \\
\hline Education & & & & .147 & 1.28 & .203 \\
\hline \multicolumn{7}{|c|}{$\begin{array}{l}\text { Dependent variable: Total Dysfunction score in PGIMS, Final Model } R=0.613, F(5,56)=6.75, p<0.001, \quad t p \\
<0.01, \neq p<0.001\end{array}$} \\
\hline
\end{tabular}

\section{DISCUSSION}

The study examined the effect of alcohol in executive functions and memory. Different domains of executive functions were impaired in $33.9 \%$ of patients with alcohol dependence Concept identification is a complex task which needs memory, deductive reasoning, problem solving and cognitive flexibility, which was impaired in most of the study participants. Similarly, deficits in programming and mental flexibility indicate towards problem in identifying and generalizing the concept and rule, planning and ability to shift and maintain a cognitive set. These findings were consistent with the proposed hypothesis that alcohol dependents are poor in identifying the concept ${ }^{8}$ and differed significantly in the task of abstract reasoning, memory discrimination

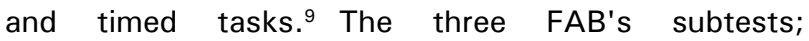
conceptualization, programming and mental flexibility which were more impaired with chronic alcohol use are found to be the tasks that have been associated with the functioning of medial, dorsolateral, and posterior areas of the prefrontal cortex. ${ }^{10}$

Duration of alcohol consumption has significant negative impact in conceptualization, programming, inhibitory control and overall executive impairments. Increase in the duration of alcohol consumption and dependence was associated with poor the executive functions. Results of the present study corroborate with previous studies ${ }^{11,12}$ highlighting the detrimental effects of alcohol in executive functions. However, executive functions were unrelated with the severity of alcohol dependence contradicting the previous studies. ${ }^{13,14}$ In these studies, level of alcohol use and blood alcohol concentration were significant predictor of cognitive impairment, including rigidity of reactions and thinking, reaction repeatability and lower behavior flexibility. Period of alcohol dependence was a significant predictor of the executive functions consistent with previous study. ${ }^{15}$ Long term use of alcohol and its effect in the central nervous system has been established, since cerebral atrophy is a common finding among alcohol dependents and is thought to be due to the toxic effect of alcohol. ${ }^{16}$

Current study revealed that immediate recall was the most intact and visual retention was the most impaired functions among alcohol dependents. Remote memory, attention and concentration, verbal retention of dissimilar pairs and visual recognition were impaired consistent with previous studies. ${ }^{5,17,18}$ Delayed recall which assesses verbal episodic memory, was impaired in the alcohol dependents.

Attention involves focusing awareness on narrowed range of stimuli or events and concentration is the ability to direct and maintain all our effort and attention on one thing for a certain period of time. Consistent with previous studies, ${ }^{19,20}$ present study revealed that attention and concentration is impaired after chronic misuse of alcohol. Ability to focus on one task at a time and sustain concentration is important prerequisite for all cognitive functions. Impaired attention and concentration associated with chronic alcohol use could explain the reason for many cognitive deficits in the study. Poor performance in one minute verbal delayed recall and 30 minute recall on the visual retention task suggests a deficit in circuits involving delayed memory. Evidence suggests that impaired long-term memory in alcohol dependents could be explained by significantly smaller both left and right hippocampal volumes. ${ }^{21}$

Moderate to severe impairment in visual memory and recognition abilities was found in the study which was supported by previous study. ${ }^{18}$ The cause of the impairment seems likely that impaired encoding or 
retrieval strategies would account for some of the nonverbal memory deficit. Inadequate organization or errors in construction, typically associated with executive dysfunction, can affect figure recall especially in the test of visual retention. Impairment in the nonverbal tasks for instance attention, visual memory and working memory indicates functional deficits in extensive brain structures as a result of alcohol consumption. ${ }^{22}$

Period of alcohol consumption was a significant predictor of memory dysfunctions in the study indicating alcohol's detrimental effects in the brain. Life time alcohol consumption was related with impairment in verbal and nonverbal working memory, suggesting a dose effect of alcohol abuse. Impairment in visual memory is moreover related with duration of alcohol use than the verbal memories ${ }^{23}$ and memory also appears to be strongly affected by acute alcohol use. ${ }^{24}$ Older people are particularly sensitive to the toxic effects of alcohol on brain and are at high risk of developing cognitive impairments ${ }^{25}$ which is supported by the study findings. Memory dysfunctions were higher with increasing age and the age was a significant predictor for the memory dysfunctions which is consistent with previous study. ${ }^{26}$

In terms of limitation, present study did not have control over the pharmacological variables which might have affected the cognitive functions. There was lack of information regarding pre-morbid cognitive functions, intellectual abilities and the motivation. Generalisability of the study findings is questionable owing to the non-probability sampling procedure. In the study, a brief battery was used for the evaluation of executive function because executive function encompasses a broad domain of cognitive functions. Despite of these limitations, the current study supports prior research demonstrating cognitive deficits related to alcohol consumption.

\section{CONCLUSIONS}

The persons with alcohol dependence show significant deficits in various aspects of the executive functions and memory. The duration of alcohol consumption is directly associated with impairment of cognitive functions, while higher education is of protective significance particularly for executive functions. Hence, routine cognitive assessment in the alcohol treatment program may be useful for the detection and assessment of the progress of these alterations, as well as for the cognitive rehabilitation and psychosocial reintegration of alcohol dependents.

\section{ACKNOWLEDGEMENTS}

We express sincere appreciation to all the participants for their cooperation in the study.

\section{REFERENCES}

1. ICD-10. Classification of Mental and Behavioral Disorders. Clinical Descriptions and Diagnostic Guidelines. World Health Organization, Geneva; 1992.

2. Parsons OA, Nixon SJ. Neurocognitive Deficits in Alcohol dependents and Social Drinkers: a continuum? Alcohol Clinical and Experimental Research. 1998;22:954-61.

3. Cabé N, Laniepce A, Ritz L, Pitel AL. Cognitive Impairments in Alcohol Dependence: From Screening to Treatment Improvements. Encephale. 2016;Jan 13. doi: 10.1016/j. encep.2015.12.012.

4. Jha M, Sinha VK. Conceptual Abilities of Alcohol Dependent Patients - An Analysis of WCST Profile. Delhi Psychiatry Journal. 2015;18:(1):77-85.

5. Noël X, Van der Linden M, Brevers D, Verbanck P. The Contribution of Executive Functions Deficits to Impaired Episodic Memory in Individuals with Alcohol use. Psychiatry Research. 2012;198(1):116-22.

6. Phelan AL. Assessment of Verbal and Nonverbal Memory and Learning in Abstinent Alcohol dependents. (Unpublished Thesis, Master of Arts in Psychology), Cleveland State University; 2013.
7. Bernardin F, Maheut-Bosser A, Paille F. Cognitive Impairments in Alcohol-Dependent Subjects. Frontiers of Psychiatry. 2014;5(78):2-6.

8. Cunha PJ, Nicastri S, de Andrade AG, Bolla KL. The Frontal Assessment Battery (FAB) Reveals Neurocognitive Dysfunction in Substance-Dependent Individuals in Distinct Executive Domains: Abstract Reasoning, Motor Programming, and Cognitive Flexibility. Addiction Behavior. 2010;35:875-881.

9. Zinn S, Stein R, Swartzwelder HS. Executive Functioning Early in Abstinence from Alcohol. Clinical and Experimental Research. 2004;28(9):1338-46

10. Dubois B, Slachevsky A, Litvan I, Pillon B. The FAB: A Frontal Assessment Battery at Bedside. Neurology. 2000; 55:1621-6.

11. Houston RJ, Derrick JL, Leonard KE, Testa M, Quigley BM, Kubiak A. Effects of Heavy Drinking on Executive Cognitive Functioning in a Community Sample. Addictive Behaviors. 2014;39:345-9.

12. Oscar-Berman M, Marinkovic, K. Alcohol: Effects on Neurobehavioral Functions and the Brain. Neuropsychology Review. 2007;17:239-57. 
13. Barani GD, Thyagarajan S. Effect of Level of Alcohol use and Personality on Cognitive Failure among Male Alcohol dependents. Journal of the Indian Academy of Applied Psychology. 2013; 39(2):182-8.

14. Domingues SCA, Mendonça JB, Laranjeira R, Nakamura-Palacios EM. Drinking and driving: A Decrease in Executive Frontal Functions in Young Drivers with High Blood Alcohol Concentration. Alcohol. 2009; 43:657-64.

15. Naim-FeilJ,FitzgeraldPB,Bradshaw JL,LubmanDI,Sheppard D. Neurocognitive Deficits, Craving, and Abstinence among Alcohol-Dependent Individuals Following Detoxification. Archives of Clinical Neuropsychology. 2014;29(1):26-37.

16. Harper C. The Neuropathology of Alcohol-Specific Brain Damage or Does Alcohol Damage the Brain? Journal of Neuropathology and Experimental Neurology. 1998;57:101-10.

17. Singh B, Manzoor A. (2012). Alcohol Consumption and Memory Performance. Indian Journal of Social Science and Organizational Behavior. 2012;1(1) 43-6.

18. Bannerjee S, Mukhopadhyay A, Shukla V. Cognitive Deterioration of Male Drug Addicts. Journal of Indian Academy of Applied Psychology. 1997; 23(1-2):13-8.

19. Paikkatt B, Akhour S, Jahan M, Singh AR. Visuospatial Constructional Ability. Visual Memory and Recognition Ability among Individuals with Chronic Alcohol Dependence in the Rey Complex Figure Test (RCFT). Neuropsychologica. 2014; 12(3):319-28.
20. Ratti MT, Bo P, Giardini A, Soragna D. Chronic Alcohol use and the Frontal lobe: Which Executive Functions are Impaired? Alcohol and Alcohol use. 2002;105 (4):276-81.

21. Zeigler D, Wang CC, Yoast RA, Dickinson BD, McCaffree MA, Robinowitz CB. The Neuro-cognitive Effects of Alcohol on Adolescents and College Students. Preventive Medicine. 2005;40(1):23-32.

22. Kopera $M$, Wojnar $M$, Brower $K$ and Szelenberger $W$. Cognitive Functions in Abstinent Alcohol-Dependent Patients. Alcohol. 2012; 46(7):665-71.

23. Lezak MD. Neuropsychological Assessment. 3rd Edition, Oxford, University Press; 1995.

24. Cunha PJ, Novaes MA. Neurocognitive Assessment in Alcohol Abuse and Dependence: Implications for Treatment. Rev Bras Psiquiatr. 2004;26:23-7.

25. Wadd S, Randall J, Thake A, Coleman. Alcohol Misuse and Cognitive Impairment in Older People: An exploratory study. Alcohol Research UK, December; 2013.

26. Fein G, Bachman L, Fisher S. Cognitive Impairments in Abstinent Alcohol dependents. Western Journal of Medicine.1990;152:531-7. 